\title{
IdeAs
}

Idées d'Amériques

$17 \mid 2021$

Villes et culture dans les Amériques

\section{Le Salon international de l'humour de Piracicaba, un laboratoire culturel innovant ancré dans la contestation politique sous le régime militaire brésilien}

o Salão internacional de humor de Piracicaba, um laboratório cultural pioneiro enraizado no protesto político sob o regime militar brasileiro

The International Humor Exhibition of Piracicaba, an innovative cultural laboratory rooted in political protest under the Brazilian military regime

Mélanie Toulhoat

\section{(2) OpenEdition}

Journals

Édition électronique

URL : https://journals.openedition.org/ideas/10053

DOI : 10.4000/ideas. 10053

ISSN : 1950-5701

Éditeur

Institut des Amériques

Référence électronique

Mélanie Toulhoat, « Le Salon international de l'humour de Piracicaba, un laboratoire culturel innovant ancré dans la contestation politique sous le régime militaire brésilien », IdeAs [En ligne], 17 | 2021, mis en ligne le 01 mars 2021, consulté le 04 juin 2021. URL : http://journals.openedition.org/ideas/10053 ; DOI : https://doi.org/10.4000/ideas.10053

Ce document a été généré automatiquement le 4 juin 2021.

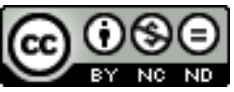

IdeAs - Idées d'Amériques est mis à disposition selon les termes de la licence Creative Commons Attribution - Pas d'Utilisation Commerciale - Pas de Modification 4.0 International. 


\section{Le Salon international de l'humour de Piracicaba, un laboratoire culturel innovant ancré dans la contestation politique sous le régime militaire brésilien}

o Salão internacional de humor de Piracicaba, um laboratório cultural pioneiro enraizado no protesto político sob o regime militar brasileiro

The International Humor Exhibition of Piracicaba, an innovative cultural laboratory rooted in political protest under the Brazilian military regime

Mélanie Toulhoat

\section{Introduction}

1 Né en 1974 dans une petite ville de l'intérieur de l'État de São Paulo, dans le sud-est du Brésil, le Salon de l'humour de Piracicaba - international à partir de sa troisième édition - fut créé à l'initiative de dessinateurs et de représentants politiques locaux. Le projet qui vit le jour sous le mandat du général Ernesto Geisel (1974-1979) consistait en la création d'un événement exclusivement dédié à la diffusion et la promotion d'un humour graphique, satirique et politique nettement inscrit dans l'opposition au régime militaire en vigueur, instauré en 1964 par un coup d'Etat militaire. La période correspond à la fin progressive des "années de plomb » au Brésil, entamées avec la promulgation en décembre 1968 de l'Acte Institutionnel $n^{\circ} 5$ (AI-5) ayant caractérisé une très nette montée en puissance de l'autoritarisme. L'année 1969 s'ouvrit en effet sur une période de suffocation des libertés individuelles et collectives, au cœur d'un durcissement brutal de l'arsenal législatif. La censure préventive des moyens de communication et d'expression fut instaurée par décret-loi le 26 janvier 1970, 
compliquant considérablement le travail de dizaines de rédactions indépendantes et contestataires, engagées dans la lutte politique, culturelle et sociale contre le régime militaire (Aquino M. A., 1999 ; Araujo M. P. N., 1999 et 2010 ; Cidreira J. H., 2017 ; Kucinski B., 1991 ; Prefeitura da Cidade do Rio de Janeiro, 2005 ; Silva M. A., 2018). Le milieu de la décennie marqua un léger infléchissement de la censure appliquée à certains journaux, mais le contrôle demeura et les pressions économiques s'accrurent. La recrudescence de la censure morale au cours de la période d'ouverture politique à partir de 1975, vint par effet de balancier compenser la progressive tolérance à l'égard de thématiques politiques à proprement parler, dans un contexte de redéfinition et de diversification de l'engagement militant à la fin des années 1970 .

Soutenu dès l'origine par certains des dessinateurs parmi les plus célèbres du pays, qui œuvraient déjà au sein de périodiques tels que Pasquim ou Opinião, le Salon qui se réunit toujours annuellement devint rapidement un lieu d'échanges, de circulations et de sociabilités à l'ampleur régionale et internationale. Gagnée en 1972 par le Mouvement Démocratique Brésilien (MDB), l'opposition tolérée et consentie par le régime, la petite municipalité de Piracicaba encouragea largement l'événement précurseur, dont l'étude permet d'appréhender la marge de manœuvre ayant existé à l'échelle municipale par rapport aux politiques culturelles menées par l'Etat fédéral. L'écrasante victoire de l'Alliance Rénovatrice et Conservatrice (ARENA) - le parti officiel soutenu par le régime militaire, seul à avoir présenté des candidats dans de nombreuses circonscriptions aux élections municipales réalisées en 1972, fut tout de même accompagnée d'une relative avancée du MDB au sein des mairies et conseils municipaux. Deux ans plus tard en revanche, les élections sénatoriales du 15 novembre 1974 furent caractérisées par un revirement crucial et inattendu : la victoire des candidats du MDB dans seize des vingtdeux Etats participant au scrutin ${ }^{1}$, qui ouvrit la perspective d'une voie légale pour faire tomber le régime. Malgré un durcissement en avril 1977 opéré par le régime pour éviter une nouvelle déconvenue dans les urnes, l'année 1974 annonça les prémices de l'ouverture politique (Napolitano M., 2011 et 2014). Nous nous proposons ici de développer une réflexion autour de l'émergence culturelle et politique, dans ce contexte politique du milieu des années 1970, du Salon de l'humour de Piracicaba sur la scène brésilienne en marge de l'axe principal du sud-est Rio-São Paulo. La petite ville, située à $164 \mathrm{~km}$ de la capitale de l'État, fut largement marquée dès la fin du XIX siècle par la fin du cycle économique caféier et la chute des prix du sucre, connaissant ensuite un certain renouveau et le développement d'infrastructures importantes au cours du second $\mathrm{xx}^{\mathrm{e}}$ siècle. La croissance démographique constitue un indice précieux de ce dynamisme : d'après les registres civils de recensement, la population passa de 152505 habitants en 1970 à 214295 en $1980^{2}$. L'événement devint un levier de transformation à partir du milieu des années 1970 pour la ville et ses habitants, marqués par l'émergence d'un festival populaire au succès incontestable, organisé autour d'un objectif à l'époque presque totalement inédit: créer un événement consacré au rire et à l'humour. Il répondit, nous le verrons, à des objectifs diversifiés allant de la contestation politique à la valorisation locale du patrimoine industriel de la ville, en passant par la professionnalisation des dessinateurs originaires de la région.

3 Les dessins primés à Piracicaba, le matériel graphique créé dans un objectif de communication et de diffusion des activités ainsi que les affiches des éditions successives révèlent une grande diversité stylistique, thématique et didactique. Ces documents iconographiques, passés à la loupe historienne, permettent de reconstituer 
des fragments d'un certain imaginaire visuel brésilien ancré dans la seconde moitié des années 1970. La répression physique, la torture, la censure et les affres du contexte politique à l'échelle nationale et internationale dominèrent largement les travaux lauréats à partir de 1974 et jusqu'en 1977. L'année suivante amorça un tournant et vit émerger de nouvelles problématiques sociales, politiques et culturelles. L'événement devenu « international » à partir de 1977 constitue un révélateur efficace de l'état du dessin d'humour brésilien de la période tout en révélant l'existence d'une considérable marge de manœuvre de l'action culturelle portée par une petite municipalité pauliste, contrainte par des enjeux économiques et sociaux, mais assumant son ancrage dans la critique du régime militaire.

\section{Faire l'histoire d'un salon de l'humour : quel terrain, quelles sources et quels objets de recherche?}

4 Notre propos et les réflexions développées dans cet article s'appuient sur les archives du Salon, consultées début 2016 au Centre National de l'Humour Graphique de Piracicaba (Centro Nacional de Documentação, Pesquisa e Divulgação de Humor Gráfico de Piracicaba - CEDHU) : documents liés à la création de l'événement, livrets de compte et bilans financiers, rapports de travail retraçant l'organisation concrète du salon, listes de participants, listes de membres des jurys, divers règlements, actes de délibération, correspondances entre les organisateurs, les autorités et les intervenants... À ces pièces manuscrites et dactylographiées, qui racontent à qui sait les lire les rouages et mécanismes internes au fonctionnement de l'événement, s'ajoutent les archives graphiques: les collections de dessins primés, les cartons d'invitation, le matériel de communication et les affiches des diverses éditions. Rappelons ici que les sources iconographiques constituent un important vivier pour analyser de nombreux aspects politiques, culturels et économiques de la société brésilienne sous le régime militaire, et des rapports de force ayant émergé et évolué entre les diverses forces sociales au fil des années. Le sociologue brésilien Marcelo Ridenti, dans son ouvrage Em busca do povo brasileiro, expliquait toute l'importance des œuvres d'art dans l'historiographie brésilienne - une réflexion pouvant largement s'appliquer aux sources issues de la production graphique humoristique : «Dans les limites du travail proposé, nous ne nous focaliserons pas à proprement parler sur la valeur intrinsèque de l'œuvre d'art, mais sur sa temporalité, en d'autres termes, l'histoire d'une société peut également être contée grâce à la production artistique ${ }^{3}$ » (Ridenti M., 2014, p. 6). En outre, confrontés aux archives de l'événement, les témoignages indirects émanant des acteurs de l'histoire du Salon, généralement retranscrits au sein d'ouvrages ou d'articles, s'avèrent également d'excellents révélateurs des circonstances de sa création, des inspirations et filiations, des difficultés et oppositions rencontrées, des enjeux de pouvoir inhérents à une telle entreprise.

5 Le travail sur le terrain à Piracicaba est intervenu assez tardivement au cours de nos recherches doctorales. Les fonds sont demeurés inaccessibles au public durant plusieurs mois pour cause de déménagement, avant d'être consultables au sein du CEDHU, une entité du Secrétariat d'action culturelle de la ville de Piracicaba, également en charge de l'organisation d'événements et d'expositions en lien avec la pratique et la promotion de l'humour graphique. Situé dans le Parc Engenho Central sur les berges de 
la rivière Piracicaba, à l'intérieur d'un bâtiment classé qui témoigne du passé industriel de la municipalitét , le centre organise toujours l'événement annuellement.

Figure $\mathrm{n}^{\circ} 1$ : Photographie du CEDHU

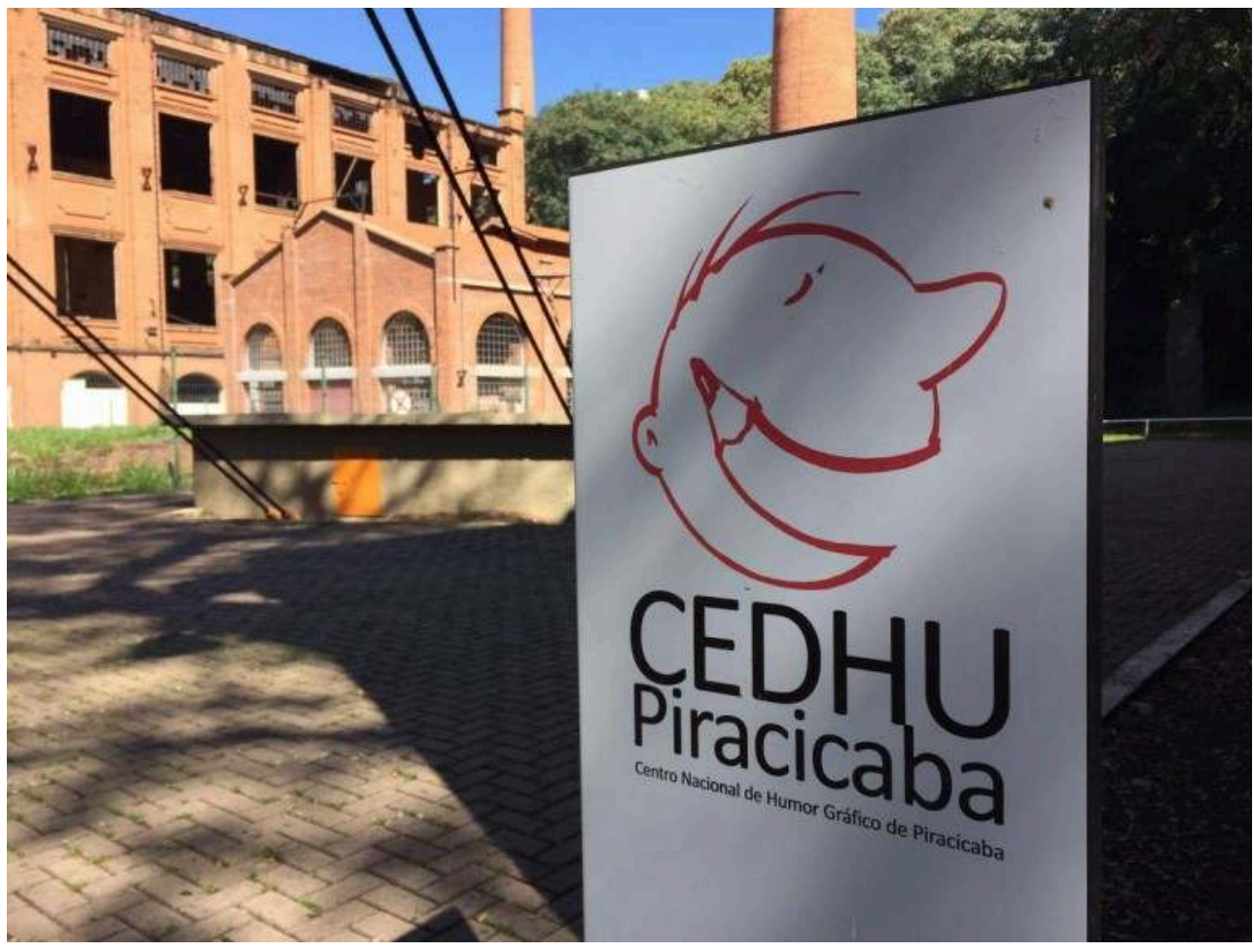

CEDHU, photographie présente sur le site internet (https://salaointernacionaldehumor.com.br/ cedhu/), page consultée le 25/01/2021

6 Le lieu chargé de l'histoire de la ville, qui comporte de nombreux bâtiments liés à l'essor de la distillerie de la canne à sucre et de la production d'alcool au XIX et début $\mathrm{xx}^{\mathrm{e}}$, classé au patrimoine de la ville et de l'État fédéré, a vécu au fil des dernières années un important phénomène de restauration et d'appropriation par les habitants. Depuis son rachat par la municipalité en 2012, le complexe de l'Engenho central abrite de nombreuses institutions et événements culturels, au premier rang desquels figurent le Salon international de l'humour et le Centre National de l'Humour Graphique de Piracicaba.

7 Il s'agit donc ici de reconstituer un exemple de politique culturelle développée par la ville de Piracicaba, à contre-courant des choix du gouvernement fédéral brésilien encore très répressif au milieu des années 1970 : rigoureuse censure appliquée aux arts et manifestations culturelles, pressions économiques, répression physique et emprisonnements. Loin de s'éteindre durant la décennie, l'humour graphique, à l'instar d'un ensemble de mécanismes et pratiques de la contestation politique, fut contraint de multiplier les stratégies de contournement des interdictions. Les marges de manœuvre de l'opposition démocratique non armée, et donc de la presse indépendante, étaient considérablement rétrécies. L'espace politique et médiatique occupé par les moyens de communications contestataires et les diverses formes d'humour et de satire politiques publiés en leur sein, était situé aux confins de la légalité et de l'illégalité, entre censuré, toléré et autorisé. Moins d'un an après la promulgation de l'AI-5 naissait à Rio de Janeiro l'hebdomadaire satirique Pasquim, qui accorda une place de choix aux formes 
visuelles de la critique des mœurs et de la satire sociale. La plupart des membres de son équipe, comme ceux de nombreuses rédactions indépendantes nées au début des années 1970, s'inséraient au sein d'une communauté militante fondée sur des valeurs et une vision du monde opposées à celles défendues par le régime, malgré une grande disparité de sympathies idéologiques, de lignes éditoriales, de styles et de pratiques artistiques. L'existence préalable de cette communauté, envisagée comme un «front politico-culturel de résistance » (Hermeto M., 2013), fut indispensable à la création du Salon de l'humour de Piracicaba.

Il convient également de replacer l'événement dans le contexte paradoxal d'une politique du régime militaire orientée vers la création d'un marché de biens culturels, alors que de nombreux artistes assumèrent leur rôle critique et d'opposition. À partir des archives d'un événement consacré à l'humour graphique initialement pensé et financé à l'échelle municipale, constitué rapidement en espace national et international de contestation politique et culturelle, nous interrogerons l'existence de marges d'action locales, révélatrices de l'existence de jeux d'échelles de la répression, de la contestation et de l'exercice des libertés collectives sous le régime militaire brésilien.

\section{Contexte municipal et acteurs du salon}

9 Les autorités municipales s'engagèrent dès les années 1950 dans d'importants projets en faveur du développement de la culture et des pratiques artistiques. En 1967, la première édition du Salon d'Art Contemporain (SAC) se tint au sein du Centre Académique Luiz de Queiroz (CALQ), une organisation étudiante représentative de l'Ecole Supérieure d'Agriculture Luiz de Queiroz (ESALQ). L'unité de l'Université de São Paulo, installée à Piracicaba et spécialisée en sciences agraires et environnementales, attira un nombre croissant d'étudiants à partir de la création du cursus de doctorat en 1970 et contribua à l'installation durable d'une population universitaire dans la ville, distante de la capitale de l'État de plus de 150 kilomètres.

10 En 1972, le professeur Ermelindo Nardim ainsi que l'ingénieur et secrétaire municipal au tourisme de Piracicaba, Luis Antonio Lopes Fagundes, décidèrent d'intégrer une exposition consacrée à l'humour graphique au sein du Salon d'Art Contemporain, désormais organisé à la pinacothèque municipale Miguel Dutra. Le journaliste Roberto Antonio Cêra, alors membre de la commission d'organisation du SAC, fut tout à fait enthousiaste à l'idée de varier les perspectives esthétiques de l'événement. L'environnement culturel et intellectuel constitué par de nombreuses institutions artistiques paulistes était propice à la naissance d'un projet voué au renforcement de l'espace occupé par l'humour graphique et l'image satirique. À cet égard, il convient de mentionner le rôle de Walter Zanini, professeur d'histoire de l'art et critique d'art, directeur du Musée d'art contemporain de l'Université de São Paulo entre 1963 et 1978. Il y développa un travail très précurseur, ouvrant un espace de relative liberté aux artistes et créateurs sous le régime militaire (Costa H., 2008 ; Freire C., 2013).

La tenue en 1973 d'un premier salon d'humour et de bande dessinée, à l'initiative de quelques étudiants de l'Université presbytérienne Mackenzie de São Paulo, constitua un autre important précédent. Cet événement antérieur, toléré au sein d'une institution d'enseignement supérieur privée et confessionnelle caractérisée par son soutien au régime dictatorial, permet de penser le rôle des divers environnements universitaires dans la constitution de projets d'initiatives contestataires, à l'instar de 
l'historien brésilien Rodrigo Patto Sá Motta. Son ouvrage As universidades e o regime militar - cultura política brasileira e modernização autoritária (Patto Sá Motta R., 2014) invite en effet à dépasser la dichotomie "répression - résistance " en tant que catégorie d'analyse du phénomène autoritaire, lui préférant le triptyque "adhésion résistance - accommodement ", davantage propice à la perception d'un ensemble de relations complexes entre les institutions, leurs organes de direction, le corps enseignant, les étudiants et les divers organes répressifs de l'Etat. Dès lors, il est possible de comprendre l'absence de censure concernant le salon d'humour et de bande dessinée de 1973, a priori complètement paradoxal dans le cas d'un événement de ce type ayant pour vocation de relier les universitaires et les différents groupes sociaux formant la communauté au sens large, et donc à ce titre particulièrement surveillé par les militaires.

Fernando Coelho dos Santos, vice-président de la Direction Centrale des Etudiants (DCE) et le dessinateur Zélio Alves Pinto, influencés par diverses expériences similaires au Canada, en Italie et en Belgique, décidèrent d'organiser un salon d'humour ouvert aux amateurs et professionnels du pays (Guimarães Aguiar Neto B. et al., 2016). De nombreux témoignages des protagonistes, publiés a posteriori, attestent une certaine relation de parenté entre le salon de l'Université Mackenzie et celui de Piracicaba, notamment fondée sur l'apprentissage tiré de l'expérience précurseure et un réseau de sociabilité entre acteurs locaux et dessinateurs d'humour politiques de São Paulo et Rio de Janeiro (CEDHU, 1993 ; Guimarães Aguiar Neto B. et al., 2016).

Adilson Benedito Maluf, élu maire en novembre 1972, apporta dès la première édition un soutien important à l'initiative du Salon de l'humour de Piracicaba. Son successeur, João Herrmann Neto, agronome de l'ESALQ, ex-président du CALQ, instaura à partir de son élection en 1977 un programme pionnier d'éducation familiale à Piracicaba. À la tête de diverses initiatives favorisant la participation des classes les plus populaires de la ville aux décisions administratives, il encouragea aussi largement le développement de l'événement.

Le groupe fondateur du salon de l'humour de Piracicaba était composé de Roberto Antônio Cêra et de ses confrères journalistes Adolfo Queiroz et Carlos Colonese, des professeurs Alceu Marozi Righetto et Ermelindo Nardim, ainsi que de Luis Antônio Lopes Fagundes. À la suite d'une première visite de Nardim et Cêra à Rio de Janeiro en 1972, pour organiser une exposition de dessins originaux à Piracicaba, la deuxième prise de contact avec les dessinateurs de l'hebdomadaire satirique carioca Pasquim eut lieu au début de l'année 1974. L'admiration pour les caricaturistes Ziraldo, Jaguar, Millôr Fernandes ou Henfil, à la renommée alors déjà bien installée, était liée à la reconnaissance de leur talent et célébrité, à la diffusion de leurs travaux, mais également à la réputation sulfureuse de membres de la bohême intellectualisée de Rio de Janeiro. Les membres de la rédaction de Pasquim acceptèrent de contribuer à la création du projet et participèrent massivement à la première édition de l'événement en 1974 et aux suivantes.

\section{Une politique culturelle inscrite dans la contestation}

L'affiche réalisée par Zélio Alves Pinto à l'occasion du premier salon de l'humour de Piracicaba en août 1974, est tout à fait révélatrice du contexte politique de production de l'image et du défi représenté par l'initiative en pleine période d'institutionnalisation 
de l'État autoritaire. Face aux craintes de censure, celui qui était devenu le parrain du projet avait opté pour un graphisme sobre, en noir et blanc, mettant en scène un personnage souriant à la tête débordant des armes du dessinateur d'humour politique : crayons, pinceaux, stylos et « $\mathrm{V} »$ de la victoire.

Figure $\mathrm{n}^{\circ} 2$ : Affiche de la première édition du Salon de l'humour de Piracicaba

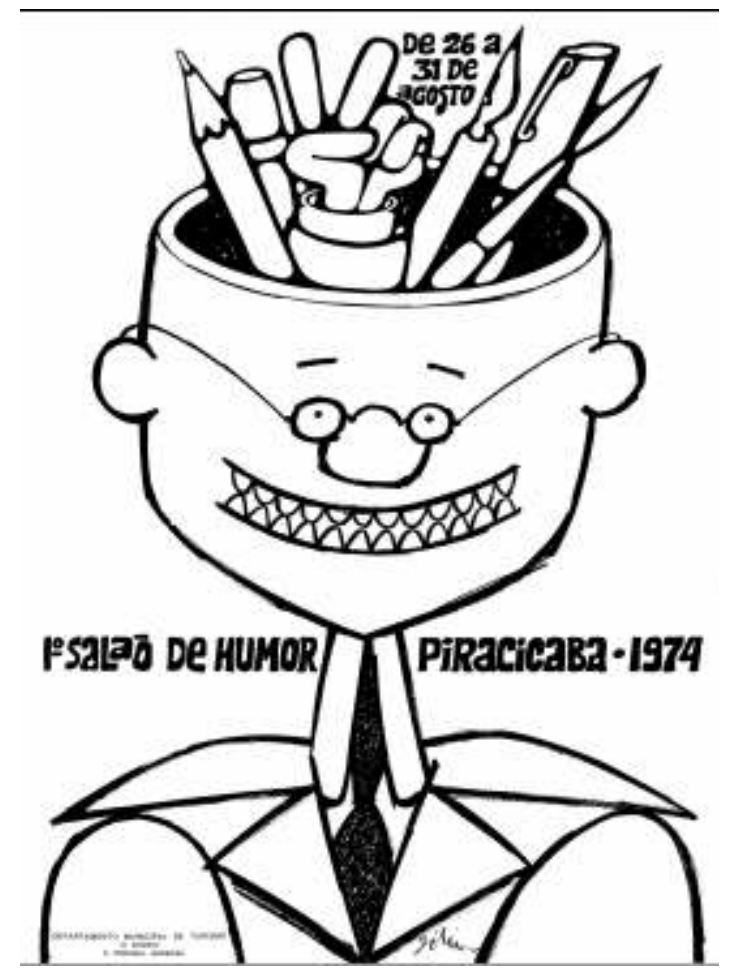

Archives du CEDHU, Piracicaba, Zélio Alves Pinto, Affiche du $7^{\text {er }}$ Salon de l'humour de Piracicaba, 1974

Le visuel mentionnait le soutien du Département municipal de tourisme de la ville, ainsi que celui du journal local $O$ Diário et du club Colonel Barbosa, un cercle de l'élite locale fondé en 1940. Les documents audiovisuels réalisés par Roberto Antônio Cêra en 1974 et $1975^{5}$ permettent de dessiner un profil du public venu aux deux premières éditions, majoritairement blanc, composé de personnes jeunes, mais également de familles et de gens plus âgés qui déambulaient dans les vidéos au milieu des galeries de panneaux classés par artiste. La prépondérance notable du public étudiant, notamment liée à la proximité de l'ESALQ et de jeunes paulistes venus en nombre, impliqua une volonté de la part des organisateurs de faire sortir l'ensemble des manifestations culturelles existant en marge du salon, du seul champ universitaire, extrêmement surveillé et censuré. La présence massive des journalistes de l'hebdomadaire Pasquim, dont la production graphique était largement ancrée depuis sa création en 1969 dans la contestation du régime autoritaire, contribua d'emblée à forger une identité critique et satirique.

La correspondance du salon permet de retracer les échanges réalisés avec des dessinateurs, plus ou moins connus et expérimentés, issus des quatre coins du pays. L'artiste originaire de Fortaleza, Hermógenes Gomes Magalhães, dit Hermó, rédigea une note manuscrite ${ }^{6}$ dans laquelle il indiquait son enthousiasme et sa volonté de participer à l'événement, malgré le retard dans l'envoi de ses travaux. L'informalité du langage et la sympathie dénotée traduisent une certaine intimité entre le président de la 
commission d'organisation du salon et le dessinateur, dont le retard dans l'envoi des dessins ne s'avéra pas être un obstacle puisqu'il remporta un prix au cours de cette première édition. Une autre lettre manuscrite, signée Maroubo et rédigée sur une page de carnet d'écolier à lignes ${ }^{7}$, fut envoyée le 19 août 1974 depuis la ville d'Assis, dans l'État de São Paulo.

Le jury de la première édition, dont la composition est détaillée dans un document de compte-rendu, était formé par les membres de la rédaction de Pasquim Millôr Fernandes, Ziraldo, Fortuna et Jaguar, ainsi que Zélio Alves Pinto. Soixante-quinze caricaturistes et dessinateurs s'inscrivirent au concours, envoyant un total de deux cent vingt travaux. Les archives du CEDHU renferment également un document manuscrit, non signé, retraçant les délibérations du jury et les préférences de chaque membre jusqu'à l'obtention d'un consensus ${ }^{8}$.

Figure $n^{\circ} 3$ : Délibérations des membres du jury du $1^{\text {er }}$ Salon de l'humour

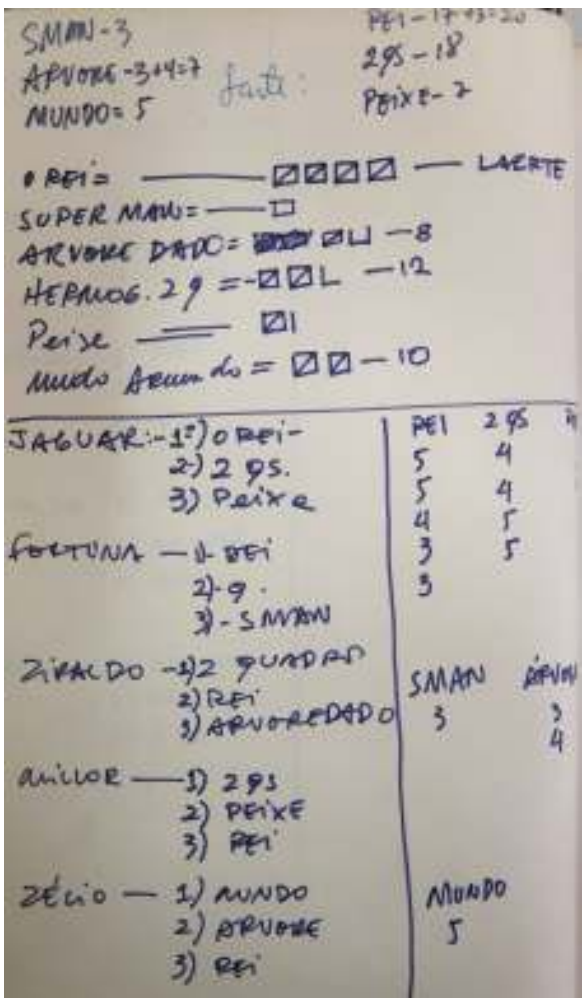

Archives du CEDHU, Piracicaba, fonds « 1974 », Document de délibérations des membres du jury pour les prix accordés aux dessins envoyés.

Les trois dessins primés lors de cette première édition dénotent un profond ancrage dans la contestation et la lutte contre la vision du monde imposée par le régime militaire, une tendance qui ne fit que se confirmer au fil des années. Le premier prix fut attribué à une création originale de la dessinatrice Laerte Coutinho, sévissant déjà au sein de Balão, un périodique édité par les étudiants de l'École de communication et arts (ECA) de la USP. Référence à la fable de Hans Christian Andersen, «Les habits neufs de l'empereur ", le dessin désigné comme « Rei » dans les délibérations du jury, abordait dès 1974 les thématiques de la répression physique et de la torture par le biais d'un vocabulaire visuel médiéval. La scène représentait un enfant victime de ses tortionnaires, suspendu par les bras et des boulets attachés aux pieds. Au premier plan, 
un bourreau encapuchonné arborait un sourire carnassier, alors qu'il chauffait à blanc une barre en métal, outil de la torture proche. En arrière-plan, un groupe impatient assistait à la scène. L'analogie entre les techniques de torture passées et contemporaines de la production de l'image prenait corps dans le mélange disparate de tenues vestimentaires et d'accessoires, pour dénoncer la brutalité de la répression physique et les violentes barrières imposées à la liberté d'expression. Contrairement aux paroles de l'enfant s'écriant dans la fable d'Andersen «Mais il ne porte pas d'habits!»- expression progressivement remplacée dans les versions et traductions successives par «Le roi est nu»!-, le protagoniste torturé dans le dessin reproduit ciaprès hurlait justement le contraire : "Le roi était vêtu!» (« 0 rei estava vestido!»). L'image satirique aux multiples niveaux de lecture, qui remporta le premier prix en 1974, dénonçait d'une part l'absurdité d'un contexte répressif au sein duquel la liberté d'expression pouvait conduire à la torture physique, et d'autre part la censure morale prohibant les représentations ou l'évocation de la nudité.

Figure $n^{\circ} 4$ : Dessin de Laerte Coutinho, lauréat du $1^{\text {er }}$ prix à Piracicaba en 1974

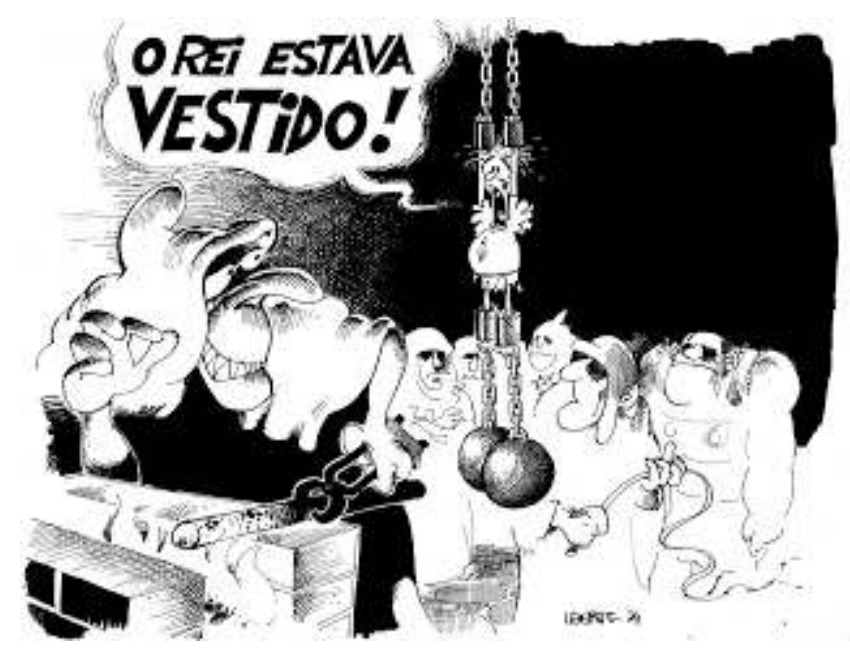

Archives du CEDHU, Piracicaba, fonds « 1974 », Laerte Coutinho, $7^{\text {er }}$ prix du Salon de l'humour de Piracicaba

Le deuxième prix fut attribué au dessinateur Hermógenes Gomes Magalhães, dit Hermó, pour une création ancrée dans le périlleux contexte de Guerre froide et l'imminence d'un conflit nucléaire au potentiel apocalyptique. Un homme suicidaire, un pistolet pointé sur la tempe gauche, était perché au sommet d'une planète chaotique perdue dans la course effrénée aux armements. L'omniprésence d'armes lourdes et de satellites, symboles du climat de Guerre froide régnant au milieu des années 1970, contrastait avec un petit îlot représenté en arrière-plan, synonyme d'une préoccupation environnementale naissante dans le contexte brésilien. L'habitant de la planète voisine assistait de loin à l'autodestruction belliqueuse au premier plan, apparemment pleinement satisfait de son foyer arboré et pacifique. 
Figure $n^{\circ} 5$ : Dessin de Hermógenes Gomes Magalhães, lauréat du $2^{\mathrm{e}}$ prix à Piracicaba en 1974

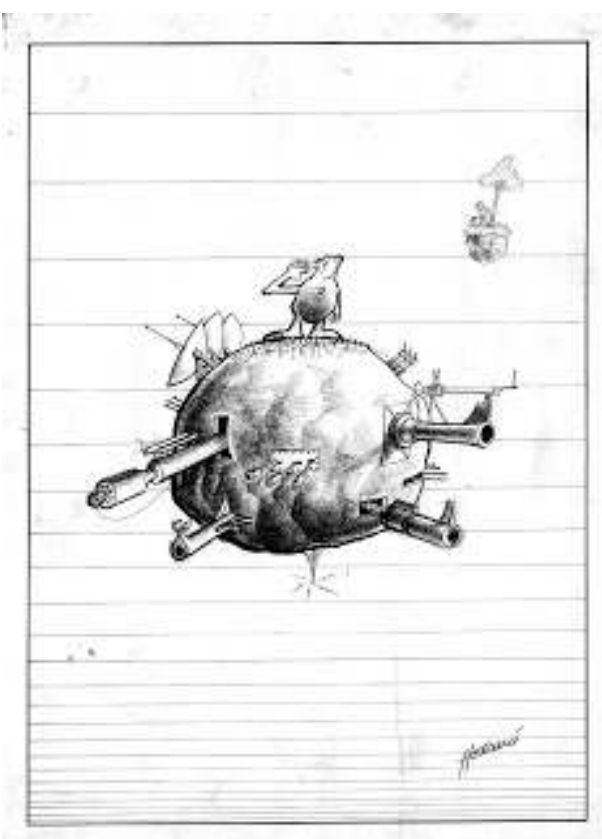

Archives du CEDHU, Piracicaba, fonds « 1974 », Hermógenes Gomes Magalhães, $2^{e}$ prix du Salon de I'humour de Piracicaba

21 Le troisième prix, enfin, revint à Luiz Renato Bittencourt, pour le dessin reproduit ciaprès, traduisant sa grande préoccupation à l'égard des zones naturelles brésiliennes et dénonçant avec poésie l'absurdité de la déforestation. Il critiquait la déforestation par le biais d'un ensemble bigarré d'oiseaux agglutinés sur un arbre isolé, isolé au milieu d'un champ de souches et que l'un des animaux tentait de réparer en fixant un manche de hache à l'une de ses branches coupées. 


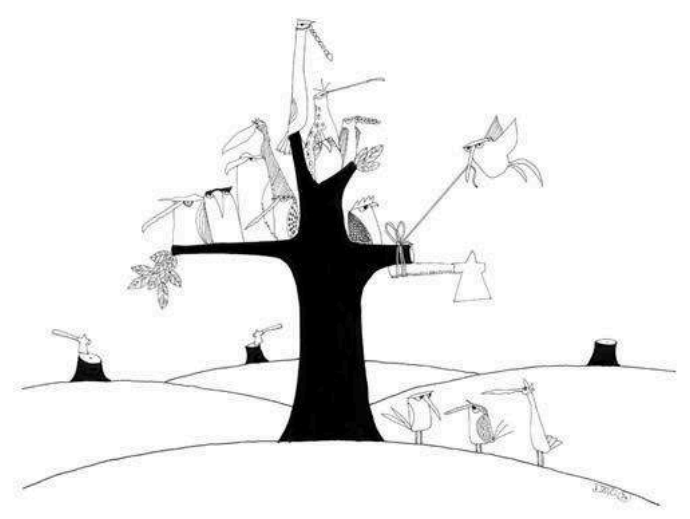

Archives du CEDHU, Piracicaba, fonds « 1974 », Luiz Renato Bittencourt, 3e prix du Salon de l'humour de Piracicaba

Le fait que deux des trois dessins primés à Piracicaba dès 1974 abordent la nécessaire préservation de l'environnement et des ressources naturelles, est révélateur d'une prise de conscience précoce des enjeux de l'écologie par les dessinateurs. Présente en filigrane au sein de divers périodiques indépendants non spécialisés au cours de la première moitié des années 1970, tels que Pasquim, Ex ou Coojornal, cette thématique devint ensuite la préoccupation centrale de nouvelles publications au cours de la seconde moitié de la décennie. Elle surgit et s'élargit considérablement, en réponse à la vision ufano-développementaliste de la dictature militaire prônant la conquête et la colonisation modernes des espaces intérieurs par les pionniers, envisageant de fait la nature comme un ensemble de ressources productives à exploiter. Citons notamment Raízes, publication du Centre d'études écologiques de la ville de Santos, qui vit le jour en juillet 1977 et annonça d'emblée ses intentions en se présentant comme un « journal de résistance écologique ${ }^{9} "$.

Cette première édition du salon, épargnée par la censure et la répression, dépassa les attentes des organisateurs. Le soutien matériel de la ville, qui finança en grande partie l'événement, et la présence de dessinateurs célèbres jouissant d'un important prestige au sein de la profession et du public amateur d'humour satirique, contribuèrent très certainement au succès de l'entreprise. Ce constat nous autorise à formuler des hypothèses sur l'importance de l'échelle locale dans la mise en œuvre et la poursuite d'un tel projet.

Les préoccupations des organisateurs se focalisèrent de plus en plus, au fil des années, sur la participation des classes populaires aux festivités. Rédigés au cours de l'année 1982, dans un contexte bien davantage marqué par l'ouverture politique qu'au moment 
de la création du Salon, plusieurs comptes-rendus internes de réunions préparatoires du comité d'organisation insistent sur la volonté de rendre l'événement plus accessible, attractif et intéressant aux yeux des habitants les plus défavorisés et pas seulement à ceux de l'élite intellectualisée de la ville. Un document recommandait notamment de renforcer la décoration thématique de l'espace urbain, en soulignant certains aspects fondamentaux :

Le Salon d'humour doit s'ouvrir à tous, avec une grande joie contagieuse dans la ville, qui est la seule du Brésil à maintenir aussi bien le salon d'humour. Il faut rechercher, à travers la publicité et une meilleure diffusion, la participation des habitants de Piracicaba en général et pas seulement d'une élite. Ont été suggérés des ballons commémoratifs, des « bouches » souriantes comme entrées de la ville, en résumé, une décoration faisant allusion au thème de l'humour et au salon ${ }^{10}$.

À n'en pas douter, les structures municipales, les ambitions de dynamisme culturel de la ville, la valorisation de partenaires économiques implantés localement et la portée initialement assez modeste du rayonnement de l'événement - qui s'avéra tout de même national dès 1974, comme l'attestent des coupures de presse issues de nombreux périodiques parus aux quatre coins du pays - ont forgé de solides bases, prêtes à l'épreuve de l'internationalisation.

\section{Changement d'échelle et circulations internationales}

27 Actuel lieu emblématique des rencontres et circulations de dessinateurs d'humour politique au Brésil, le Salon de l'humour de Piracicaba s'est construit et forgé progressivement en tant qu'espace culturel, politique et professionnel à l'ampleur nationale et internationale. Dès la fin de l'année 1974, la Commission organisatrice envisagea d'étendre le spectre de son action et invita l'année suivante des personnalités célèbres hors des frontières brésiliennes. Voué à la diffusion et à l'exposition de l'humour graphique, l'événement constitua également un lieu de sociabilités et de débats en pleine période répressive. Claude Moliterni, alors directeur de la revue Phénix et directeur éditorial chez Dargaud, l'un des créateurs du Salon

international de la bande dessinée d'Angoulême dont la première édition fut organisée le 25 janvier 1974, avait rencontré Zélio Alves Pinto à Paris au cours de la décennie précédente. Zélio était parti en France dans les années 1960 pour étudier la peinture et organisa sa première exposition individuelle à la Maison du Brésil en 1962. Il servit d'intermédiaire entre les organisateurs du salon de Piracicaba et Moliterni. Luis Antônio Fagundes, directeur du département de tourisme de Piracicaba, adressa le 22 décembre 1974 à l'éditeur français une lettre qui atteste les échanges préalables ayant existé entre les organisateurs des salons respectifs :

Nous aimerions maintenant savoir combien d'originaux vous voudriez exposer au salon de Piracicaba. Il est important que nous le sachions, puisque nous allons maintenant nous occuper du transport de ces travaux, dans la mesure où nous souhaitons que l'exposition des originaux des artistes de la maison d'édition Dargaud soit une attraction dans tous les sens du terme. Nous aimerions que les originaux des artistes de Dargaud nous soient envoyés avant votre venue, de cette manière nous aurons plus de temps pour traiter le matériel qui sera exposé. L'idéal serait que les originaux des artistes de Dargaud arrivent à Piracicaba au plus tard la dernière semaine du mois de juillet ${ }^{11}$. 
fonds de lettres envoyées et reçues, classés par année, permettent de retracer les étapes d'organisation de la venue de Moliterni à Piracicaba en 1975, ainsi que les prémices de l'internationalisation de l'événement. En juillet 1975, Rogério Otávio Viana, directeur des relations publiques de la ville de Piracicaba, écrivait aux directeurs du Salon d'Angoulême pour leur demander de bien vouloir diffuser un appel à participation au sein de leurs réseaux d'artistes et dessinateurs ${ }^{12}$. Ce courrier indique bien la nécessité de faire connaitre le projet à l'étranger. Entre juin et août 1975, de nombreux articles consacrés au caractère exceptionnel de la présence de Moliterni parurent dans la presse locale piracicabense. Les journalistes insistèrent dans leur grande majorité sur les possibilités, découlant de l'événement, d'insertion dans une sorte de marché européen de travaux brésiliens complètement idéalisé, une perspective présentée comme susceptible d'attirer les professionnels du secteur venus depuis le pays tout entier ${ }^{13}$.

L'année suivante, ce fut au tour de Geoffrey Dickinson, de la revue satirique londonienne Punch, et de Sérgio Aragones, travaillant au sein du déjà célèbre magazine nord-américain Mad, de venir à Piracicaba. Aux côtés du dessinateur argentin Hermenegildo Sabát, de l'éditrice brésilienne Sonia Hirsch Duncan et du journaliste brésilien Mino Carta, ils intégrèrent le jury de la troisième édition du salon. Dans ce cas également, la correspondance abondante détaille les étapes des échanges et de la concrétisation des invitations, retraçant également l'organisation d'une exposition de dessins issus des deux publications anglo-saxonnes, transportés depuis l'Angleterre. Les documents de travail montrent une préoccupation croissante de bilinguisme dans l'impression des programmes et supports de communication, ainsi que l'indispensable présence d'interprètes, autant d'indices supplémentaires d'un souci d'ouverture sur le monde du cartoon professionnel. Dépassant le cadre local et municipal, les organisateurs invitèrent pour la première fois au cocktail d'inauguration de 1976 des personnalités politiques de premier plan, telles que Ney Braga, alors Ministre de l'Éducation du gouvernement Geisel, Olavo Egydio Setubal, maire de la ville de São Paulo, ou encore Max Pfeffer, directeur du Département de Culture, Science et Technologie de l'État de São Paulo. La liste des cent deux dessinateurs sélectionnés pour participer à la compétition indique la présence exclusive de brésiliens, venus de tout le pays, à l'exception de l'argentin Oscar Grillo.

À la suite d'un premier moment, de 1974 à 1976, caractérisé par la survalorisation de célèbres productions anglo-saxonnes et européennes, les organisateurs se tournèrent vers le marché éditorial national et surtout, régional latino-américain, devant affronter la difficile concurrence des gibis nord-américains ${ }^{14}$. Dès lors, l'année 1977 marqua en ce sens un tournant majeur dans la définition de ce caractère international : les organisateurs invitèrent et rendirent davantage hommage aux dessinateurs latinoaméricains dans une logique d'affranchissement progressif du dessin d'humour vis-àvis des grandes célébrités occidentales. Le contexte politique de la seconde moitié des années 1970, profondément marqué par l'instauration de régimes autoritaires dans les pays voisins, amena le Salon de Piracicaba à se constituer en espace de rencontre et sociabilité entre confrères latino-américains. Le périodique Tribuna da Imprensa commenta le 26 août 1977 ce tournant majeur dans les intentions des organisateurs, tangible à partir de la quatrième édition :

Cette année, pas seulement pour des raisons économiques, la présence des grandes vedettes étrangères qui animèrent beaucoup les salons antérieurs, mais contribuèrent peu à ces derniers (Claude Moliterni, Sérgio Aragones, Geoffrey 
Dickinson) fut évitée. De cette manière, la préoccupation presque colonisée de vouloir obtenir pour le salon l'approbation de nord-américains et d'européens fut dispensée. Les deux artistes étrangers présents cette année furent les dessinateurs argentins Fontanarrosa et Crist, qui sont venus par leurs propres moyens, invités par la revue Boca de São Paulo ${ }^{15}$.

D'autre part, les comptes-rendus successifs des différentes éditions permettent de mesurer précisément l'ouverture aux dessinateurs latino-américains et de chiffrer cette évolution. Parmi les cinquante candidats sélectionnés pour le «Prix du dessin de presse de 1978 » figuraient le finlandais Kimmo Rompotti, l'espagnol Humberto de la Torre Quevedo, l'argentin Marcelo Lawryczenko, le salvadorien José Bernardo Pacheco, le colombien Wilfrido Ramirez et le portugais Antonio Moreira Antunes. Cette année-là, les récompenses furent attribuées à cinq dessinateurs brésiliens, Luiz Osvaldo Rodrigues, dit LOR, Francisco Juska Filho, Glauco Villas Boas, Fausto Guilherme Longe et Josanildo Dias de Lacerda, ainsi que l'argentin Crist venu au salon l'année précédente. En 1982, plusieurs dessins furent pour la première fois envoyés depuis l'étranger pour le concours général et parmi les quatre-vingt-trois artistes sélectionnés pour l'exposition figuraient quatre argentins, trois colombiens, un péruvien, un costaricain, un guatémaltèque et un belge. Cette tendance à l'internationalisation, initialement timide, et la reconnaissance croissante de l'événement à l'étranger, se poursuivirent jusqu'aux prémices de la transition démocratique et la fin du régime militaire, qui marquèrent le début de nouvelles évolutions, davantage thématiques et stylistiques. Un document de présentation daté de 1985 fait état de sept objectifs majeurs ayant motivé l'organisation de cette douzième édition :

1- Valoriser les arts graphiques et le dessin d'humour au Brésil, en cherchant à lui conférer le même niveau d'importance que les arts plastiques.

2- Promouvoir une rénovation dans le champ des arts graphiques, en permettant le surgissement de nouveaux noms.

3- Rendre plus connu le travail d'artistes graphiques nationaux et étrangers à la valeur reconnue.

4- Stimuler l'échange avec des artistes et des professionnels d'autres pays.

5- Promouvoir le débat et les discussions au sujet de la condition professionnelle du dessinateur.

6- Établir un espace d'union et d'harmonie entre les artistes graphiques, pour une meilleure reconnaissance de la classe.

38 7- Délocaliser dans une ville de province les manifestations culturelles qui n'auraient leur place que dans l'axe Rio-São Paulo ${ }^{16}$.

Notons l'importance de ce dernier point, explicitement formulé par les organisateurs du Salon conscients de la nécessaire création d'un pôle artistique pérenne en marge de " l'axe Rio-São Paulo " afin de dynamiser la vie culturelle locale. Les troisième et quatrième points insistaient sur la vocation émergente du salon à favoriser les échanges de pratiques, les circulations d'expériences et de connaissances entre dessinateurs brésiliens et étrangers, a fortiori latino-américains, à l'aube du retour à la démocratie. Les enjeux du Salon présents en filigrane depuis plusieurs années semblaient également largement s'orienter vers la légitimation de l'humour graphique au sein des arts plastiques, la valorisation d'un statut professionnel pour les artistes 
nationaux ainsi que la protection de la jeune création artistique locale et régionale sur le marché du travail.

\section{Considérations finales}

Objet d'étude inédit et protéiforme, le Salon international de l'humour de Piracicaba s'ancra durablement dans le paysage culturel régional, élargissant un peu plus l'espace légal borné par la censure et la clandestinité. Observatoire à l'échelle municipale des prémices de la transition politique amorcée au cours de la seconde moitié des années 1970, le salon contribua à diffuser certaines thématiques innovantes et contestataires émergentes au sein de l'opposition politique.

Les archives du salon dévoilent un ensemble d'enjeux politiques, économiques, culturels et esthétiques caractéristiques de cet observatoire de la contestation par l'humour graphique, à l'ancrage local indéniable, dont les affiches successives et dessins primés au fil des ans permettent d'appréhender des évolutions thématiques et stylistiques majeures, ainsi que les continuités et ruptures dans le traitement de certains sujets d'ordre politique, économique et social liés à la situation vécue par la population brésilienne au cours de la seconde moitié des années 1970.

Au début des années 1980, de nouvelles thématiques inexistantes jusqu'alors firent leur entrée dans les dessins primés, et l'engagement politique caractéristique du Salon international de l'humour de Piracicaba changea progressivement de forme. Dans un contexte marqué par les grandes grèves et le développement du mouvement syndical brésilien autour du pôle urbain de São Paulo, le monde du travail et les relations de pouvoir entre ouvriers et patrons acquirent une importante visibilité. De même, les sources visuelles issues de la période de l'ouverture révèlent les inquiétudes liées au futur d'un pays à reconstruire et à la polarisation politique caractéristique du début des années 1980, autant d'enjeux dont les débats, les dessinateurs, les affiches et les dessins de Piracicaba furent à la fois témoins et acteurs.

\section{BIBLIOGRAPHIE}

Aquino, Maria Aparecida de, Censura, imprensa, Estado autoritário (1968-1978): o exercício cotidiano da dominação e da resistência, O Estado de São Paulo e Movimento, Bauru, EDUSC, 1999.

Araujo, Maria Paula Nascimento, «Imprensa Alternativa », Abreu, Alzira Alves de et al. (dir.), Dicionário Histórico-Biográfico Brasileiro - Pós 30, Rio de Janeiro, CPDOC, 2010, en ligne : http:// www.fgv.br/cpdoc/acervo/dicionarios/verbete-tematico/imprensa-alternativa (consulté le 04/02/2021).

Araujo, Maria Paula Nascimento, «Uma história do tempo presente: política, esquerda e imprensa alternativa no Brasil dos anos 70 », Carvalho Costa, Luiz Flávio, Moreira, Roberto José, Bruno, Regina, Mundo Rural e Tempo Presente, Rio de Janeiro, Mauad, 1999, p. 149-170. 
CEDHU, O I Salão Mackenzie de humor e quadrinhos e sua relação com o salão internacional de humor de Piracicaba 1973.1993, Piracicaba, Engenho central, 1993.

CEDHU, Piracicaba 30 anos de humor, São Paulo, Imprensa Oficial do Estado, 2003.

Cidreira, Jefferson Henrique, « Mídia culturais: figurações da resistência no jornal Varadouro e no cinema em Rio Branco, de 1977 a 1981 », Revista de História, vol. 6, n 1-2, 2017, p. 156-171.

Costa, Helouise, « Da fotografia como arte à arte como fotografia: a experiência do Museu de Arte Contemporânea da USP na década de 1970 ", Anais do Museu Paulista: História e Cultura Material, vol. 16, nº 2, juil.-déc. 2008, p. 131-173.

Freire, Cristina, Walter Zanini: escrituras críticas, São Paulo, Annablume, 2013.

Guimarães Aguiar Neto, Benedito, Dos Santos, Gabriel Ferrato, Guimarães, Alexandre Huady Torres et al., 1973 - Quando tudo começou - História do I Salão Brasileiro de Humor e Quadrinhos, São Paulo, Editora EME/Nova Consciência, 2016.

Hermeto, Miriam, « Grupo Casa Grande (1974-1979). Uma frente político-cultural de resistência », Napolitano, Marcos, Czajka, Rodrigo, Patto Sá Motta, Rodrigo (dir.), Comunistas brasileiros. Cultura política e produção cultural, Belo Horizonte, Editora da UFMG, 2013, p. 293-315.

Kucinski, Bernardo, Jornalistas e Revolucionários. Nos tempos da imprensa alternativa, São Paulo, Scrita Editorial, 1991.

Napolitano, Marcos, 1964. História do Regime Militar Brasileiro, São Paulo, Editora Contexto, 2014.

Napolitano, Marcos, « O golpe de 1964 e o regime militar brasileiro. Apontamentos para uma revisão historiográfica », Historia y problemas del siglo XX, vol. 2, 2011, p. 209-217.

Patto Sá Motta, Rodrigo, As universidades e o regime militar: cultura política brasileira e modernização autoritária, Rio de Janeiro, Zahar, 2014.

Prefeitura da Cidade do Rio de Janeiro, Imprensa Alternativa. Apogeu, queda e novos caminhos, Rio de Janeiro, Prefeitura, 2005.

Queiroz, Adolpho, Vicente, Evaldo, Ciasi, Letícia Hernandez, Salão internacional de humor de Piracicaba, São Paulo, Riopedrense Ltda, 2014.

Ridenti, Marcelo, Em busca do povo brasileiro. Artistas da revolução, do CPC à era da TV, $2^{\mathrm{e}}$ éd., São Paulo, Editora UNESP, 2014, p. 6.

Sarruge Molina, Rodrigo, « História do movimento estudantil: centro acadêmico da ESALQ/USP de 1909 a 2016 », Argumentos Pró-Educação, vol. 2, n 5, 2017, p. 210-234.

Silva, Marcos Antônio da, Rir das ditaduras - os Dentes de Henfil (Fradim - 1971/1980), São Paulo, Intermeios, 2018.

\section{NOTES}

1. Jornal do Senado, édition spéciale, $n^{\circ} 4207$, Ano XX, 19/11/2014, en ligne : https:// www12.senado.leg.br/jornal/edicoes/especiais/2014/11/19/jornal.pdf page consultée le $04 / 02 / 2021$.

2. Instituto de Pesquisa e Planejamento de Piracicaba - IPPLAP, «Estimativa, contagem e população censitária do munícipio de Piracicaba - 1872 a 2020 » file://C:/Users/M\%C3\%A9lanie/ Downloads/Estimativa\%20Populacional\%20do\%20Municipio\%20-\%201872\%20a\%202020.pdf, page consultée le 20/09/2020. 
3. Toutes les traductions du portugais du Brésil vers le français sont de l'autrice de cet article.

4. Le CEDHU se situe au 454, avenue Maurice Allain (13 405-123 Piracicaba, dans l'Etat de São Paulo).

5. Roberto Antônio Cêra, «Salões de Humor de Piracicaba » 1974 1975, document audiovisuel, archives privées, https://www.youtube.com/watch?v=HDFc7vHrB2Y , page consultée le 28/09/2020.

6. Archives du CEDHU, Piracicaba, fonds «Correspondance 1974 - Lettres reçues », Lettre manuscrite de Hermógenes Gomes Magalhães (Hermó) à Alceu Marozi Righeto, Piracicaba, non daté.

7. Archives du CEDHU, Piracicaba, fonds "Correspondance 1974 - Lettres reçues ", Lettre manuscrite de José Gonçalves Maroubo (Maroubo) aux organisateurs du Salon de l'humour, Assis, 19/08/1974.

8. Archives du CEDHU, Piracicaba, fonds « 1974 », Document de délibérations des membres du jury pour les prix accordés aux dessins envoyés.

9. Raízes, n¹, 07/1977, p. 1.

10. Archives du CEDHU, Piracicaba, fonds « 1982 », document « 1ra REUNIÃO ».

11. Archives du CEDHU, Piracicaba, Fonds «Correspondance 1974 - Lettres envoyées », Lettre de Luis Antônio Fagundes à Claude Moliterni, Piracicaba, 22/12/1974.

12. Archives du CEDHU, Piracicaba, Fonds « Correspondance 1975 - Lettres envoyées », Lettre de Rogério Otávio Basílio Viana aux organisateurs du Salon international de la bande dessinée d'Angoulême, Piracicaba, 8/07/1975.

13. Archives du CEDHU, Piracicaba, Fonds «Revue de presse 1975 », coupure de O Diário, 18/06/1975.

14. «Opções e lutas do quadrinho nacional », Movimento, $n^{\circ} 103,20 / 06 / 1977$, p. 14.

15. Archives du CEDHU, Piracicaba, Fonds «Revue de presse 1977 », coupure de Tribuna da Imprensa, 26/08/1977.

16. Archives du CEDHU, Piracicaba, fonds « 1985 », document de présentation «XII SALÃO INTERNACIONAL DE HUMOR DE PIRACICABA ».

\section{RÉSUMÉS}

Né en 1974 en pleine période répressive sous le régime militaire brésilien, le Salon de l'humour de Piracicaba, devenu «international » en 1976, fut créé à l'initiative de dessinateurs et représentants de la petite municipalité pauliste située en marge de l'axe culturel majeur du sudest brésilien « Rio de Janeiro-São Paulo ». Entièrement voué à la création, la promotion et la diffusion de l'humour graphique, l'événement largement ancré dans la contestation de l'autoritarisme répondit à différents enjeux majeurs au fil des années : dynamisme culturel et économique à l'échelle locale, valorisation de la production graphique nationale, circulations des caricaturistes latino-américains et professionnalisation, lutte contre la censure et les pressions émanant de l'Etat fédéral, enjeux liés à son internationalisation. Largement encouragé et financé par la petite ville de Piracicaba, l'événement précurseur révèle une certaine marge de manœuvre existant à l'échelle municipale, contrastant avec la politique répressive menée par le régime militaire brésilien. Cet article se base sur l'analyse des archives de l'événement, conservées au sein du Centre National de l'Humour Graphique de Piracicaba (CEDHU), qui renferment des fonds 
à l'importance capitale afin de reconstituer des fragments de l'imaginaire visuel brésilien ancré dans la seconde moitié des années 1970.

Nascido em 1974 em meio ao período repressivo do regime militar brasileiro, o Salão de Humor de Piracicaba, que se tornou "internacional" em 1976, foi criado pela iniciativa de cartunistas locais e representantes da municipalidade paulista, na margem do grande eixo cultural do sudeste brasileiro "Rio de Janeiro-São Paulo". Inteiramente dedicado à criação, à promoção e à divulgação do humor gráfico, o evento, em grande parte enraizado no protesto contra o autoritarismo, respondeu a vários desafíos ao longo dos anos: dinamismo cultural e econômico ao nível local, valorização da produção gráfica nacional, circulação dos cartunistas latinoamericanos e profissionalização, luta contra a censura e as pressões do estado federal, assim como a sua própria internacionalização. Amplamente incentivado e financiado pela pequena cidade de Piracicaba, o evento precursor revelou a existência de uma certa margem de manobra em escala municipal, em contraste com a política repressiva do regime militar brasileiro. Este artigo é baseado na análise dos arquivos do evento, mantidos no Centro Nacional de Humor Gráfico de Piracicaba (CEDHU), que contêm fundos de uma importância capital para reconstruir os fragmentos do imaginário visual brasileiro, enraizado na segunda metade dos anos 70 .

Born in 1974 during the repressive period of the Brazilian military regime, the Humor Exhibition of Piracicaba, which became "International" in 1976, was created on the initiative of local cartoonists and municipal representatives, in the small town located on the margins of the major cultural axis of South-East Brazil "Rio de Janeiro-São Paulo". Entirely dedicated to the creation, promotion and dissemination of graphic humour, the event, which is largely rooted in the contestation of authoritarianism, has responded to various major issues over the years: cultural and economic dynamism on a local scale, valorisation of national graphic production, circulation of Latin American cartoonists and professionalisation, fight against censorship and pressure from the federal state and internationalisation. Widely encouraged and financed by the small town of Piracicaba, the precursory event revealed certain possibilities of action existing on a municipal scale, in contrast with the repressive policy carried out by the Brazilian military regime. This article is based on an analysis of the archives of the event, kept at the National Centre of Graphic Humour in Piracicaba, which contain funds of capital importance in order to reconstruct fragments of the Brazilian visual imagination rooted in the second half of the 1970s.

\section{INDEX}

Mots-clés : Brésil, Salon international de Piracicaba, régime militaire, humour graphique, caricature.

Palavras-chave : Brasil, Salão internacional de Piracicaba, regime militar, humor gráfico, caricatura.

Keywords : Brazil, International Exhibition of Piracicaba, Military Regime, Grafic Humor, Caricature.

\section{AUTEUR}

\section{MÉLANIE TOULHOAT}

Post-doctorante au sein du LabEx HASTEC de l'Ecole pratique des Hautes études (EPHE) affiliée à l'IMAF en 2020-2021, Mélanie Toulhoat est docteure en histoire de l'Université Sorbonne Nouvelle (IHEAL-CREDA) et de l'Université de São Paulo, chargée de cours à l'IHEAL 
("L'Amérique latine moderne et contemporaine : histoire et historiographie »), et viceprésidente de l'Association pour la recherche sur le Brésil en Europe (ARBRE). Contact : melanie.toulhoat@wanadoo.fr ; melanie.toulhoat@sorbonne-nouvelle.fr 